\title{
ADAPTIVE SUB-CHANNEL ALLOCATION BASED UEP FOR VIDEO TRANSMISSION IN SPACE-TIME CODED OFDM SYSTEMS
}

\author{
Guang-Hua Yang, Dongxu Shen, Victor O.K. Li \\ Department of Electrical and Electronic Engineering \\ The University of Hong Kong, Pokfulam Road, Hong Kong, China \\ Email: \{ghyang, dxshen, vli\}@eee.hku.hk
}

\begin{abstract}
In this work, we introduce the idea of adaptive sub-channel allocation based unequal error protection (ASCA-UEP) to a space-time block coded orthogonal frequency division multiplexing (STBC-OFDM) system. In such a system, UEP is realized by adaptively allocating and transmitting high-priority and low-priority video data over high-quality and low-quality sub-channels, respectively. Further, we propose two ASCA-UEP schemes in a time division duplex (TDD) system: a receiver-based scheme and a transmitter-based scheme. Analysis and simulation results demonstrate that ASCA-UEP greatly enhances the quality of video reception, and the transmitter-based scheme is more robust to uplink channel noise than the receiver-based scheme, and is thus preferred when the receiver is powerconstrained and the transmitter has sufficient power.
\end{abstract}

\section{INTRODUCTION}

The main challenge in providing reliable wireless video services is to efficiently transmit high-rate error-sensitive video data over error-prone wireless channels. Space time coded orthogonal frequency division multiplexing (STCOFDM) [1] is particularly suitable for high data rate transmission, while the combination of layered video coding with unequal error protection (UEP) can further improve the errorresistance of the compressed video [2].

In a multi-carrier system, such as OFDM, a natural way to implement UEP is based on forward error correction (FEC) [3]. By employing different channel coding schemes, video data of high-priority (HP) is given more protection than low-priority (LP) data. In such an FEC-based UEP scheme, channel knowledge is not exploited and data of different priorities are allocated to the fixed set of sub-channels for transmission. However, the multipath channel introduces uneven fading on sub-channels of OFDM symbols. HP data transmitted on sub-channels with deep fades will suffer high probability of error, even with the protection of powerful coding.

In [4], a more efficient UEP method, adaptive sub-channel allocation based UEP (ASCA-UEP), is presented for a spacetime coded OFDM system. By exploiting the channel knowledge, sub-channels are classified into high-quality (HQ) and low-quality (LQ) groups, and then used for transmitting HP and LP video data, respectively. This helps to decrease the error probability of HP data, which leads to quality improvement of the reconstructed video.

In this work, we extend the idea in [4] by exploiting the reciprocity of uplink and downlink in a time division duplex (TDD) system. Two particular ASCA-UEP schemes are proposed. One is a receiver-based scheme, in which the channel estimation and sub-channel classification are performed on the receiver and the classification results are then fed back to the transmitter for data allocation. The other is a transmitterbased scheme, in which the sub-channel classification is performed at the transmitter based on the pilot sequence sent by the receiver. Analysis and simulation results demonstrate that both schemes have almost the same performance under ideal situations. In practical situations, the transmitter-based scheme is more robust to uplink channel noise than the receiver-based scheme, and is preferred when the receiver is power-constrained and the transmitter has sufficient power.

This paper is organized as follows. In Section II, we provide the background on layered video coding, UEP, and STBC-OFDM. In Section III, we describe the ASCAUEP scheme, present the sub-channel classification method and propose the receiver-based and the transmitter-based schemes. In Section IV, we provide simulation results. Conclusion is given in Section V.

\section{BACKGROUND}

\section{A. UEP and Data Partitioning}

In current video coding standards, due to the employment of hierarchical structure and variable length coding (VLC), different portions of compressed video data have different degree of importance. For example, in a compressed MPEG2 [5] video frame, headers of each level in the hierarchical structure carry critical information and are used for synchronization. Errors in the header will render the corresponding level undecodable. On the other hand, VLCs of the discrete cosine transform (DCT) coefficients are also sensitive to errors. One bit error in a VLC may cause the loss of synchronization, which leads to undecodable bit strings until the synchronization marker carried by the next header is found. In addition, low-order VLCs are more important to the final video quality than the high-order ones.

The above properties make UEP suitable for video transmission in error-prone environments. Basically, UEP changes the distribution of errors without incurring extra 
resource consumption. In other words, less bit errors are suffered by more important data, and vice versa. To achieve UEP, layered video coding is needed to divide the video data into two or more layers with different priorities.

Data partitioning is the simplest form of layered video coding schemes. After partitioning, the bit stream from a single-layer encoder is divided into two (or more) layers, say, HP and LP layer. The HP layer carries the vital data, such as all the headers, motion vectors, and low-order VLCs; the remaining high-order VLCs and redundant copies of certain headers are carried by the LP layer. The data rate ratio of the two layers can be controlled by adjusting the number of VLCs partitioned into each layer.

The HP layer data can be decoded independently to reconstruct the video with acceptable quality. Errors in the HP layer have detrimental effects on the reconstructed video and should thus be avoided as much as possible. The LP layer data is used to improve the video quality and can tolerate more errors. Therefore, UEP aims to provide the best possible protection to the HP layer to optimize video quality. In this work, MPEG-2 with data partitioning is employed.

\section{B. Space-time block coded OFDM system}

Space-time coding (STC) achieves performance gain through transmit diversity. An important type of STC is the space-time block code (STBC), which is proposed by Alamouti [6] and generalized by Tarokh et al. [7]. The employment of STBC requires the channel to be flat. Thus, OFDM is particularly suitable for employing STBC over broadband frequency selective fading channels. Here we use Alamouti's scheme to explain the principles of STBCOFDM. For simplicity we just consider the processing on a particular sub-channel and thus omit the sub-channel index.

STBC is performed on two consecutive symbols. At the first time slot, $s_{1}$ and $s_{2}$ are transmitted simultaneously from the two transmit antennas; at the next time slot, $-s_{1}^{*}$ and $s_{2}^{*}$ (where $*$ represents the complex conjugate operation) are transmitted from the two antennas.

Assuming the channel response is constant during the two time slots, at the receiver, the baseband received signal at the two time slots can be expressed as

$$
\begin{aligned}
& r_{1}=H_{1} s_{1}+H_{2} s_{2}+n_{1} \\
& r_{2}=-H_{1} s_{2}^{*}+H_{2} s_{1}^{*}+n_{2},
\end{aligned}
$$

where $H_{1}$ and $H_{2}$ are channel responses of the two uncorrelated sub-channels, $n_{1}$ and $n_{2}$ are complex additive white Gaussian noise (AWGN) with zero mean and variance $\sigma^{2}$. Given $H_{1}$ and $H_{2}$, the decision variable for $s_{1}$ is

$$
\begin{aligned}
\hat{s}_{1} & =H_{1}^{*} r_{1}+H_{2} r_{2}^{*} \\
& =\left(\left|H_{1}\right|^{2}+\left|H_{2}\right|^{2}\right) \cdot s_{1}+H_{1}^{*} n_{1}+H_{2} n_{2}^{*} .
\end{aligned}
$$

Maximum likelihood detection is performed to get $\hat{s}_{1}$. The processing for $s_{2}$ is similar to that of $s_{1}$ and is thus omitted.

\section{ASCA-UEP}

In this section, we describe the ASCA-UEP scheme, present the sub-channel classification method and propose the receiver-based and transmitter-based schemes.

\section{A. Sub-channel Classification}

In (2), $\left(\left|H_{1}\right|^{2}+\left|H_{2}\right|^{2}\right) \cdot s_{1}$ can be viewed as the signal part, and $H_{1}^{*} n_{1}+H_{2} n_{2}^{*}$ the noise part. We define $X=$ $\left|H_{1}\right|^{2}+\left|H_{2}\right|^{2}$ as the composite sub-channel response. Let $E_{H}=E\left[\left|H_{1}\right|^{2}\right]=E\left[\left|H_{2}\right|^{2}\right]$ be the channel power, and $E_{s}$ be the average power of data symbols. Then the instantaneous power of the signal part and noise part of $\hat{s}_{1}$ can be represented as $X^{2} \cdot E_{s}$ and $X \cdot \sigma_{n}^{2}$, respectively. The instantaneous SNR can be expressed as

$$
\gamma=\frac{X \cdot E_{s}}{\sigma^{2}}
$$

From (3), we can see that the SNR of each sub-channel is determined by $X$. Thus, if the same modulation and channel coding scheme is employed on all the sub-channels, the bit error rate (BER) of each sub-channel is only determined by the composite sub-channel response $X$. Then the subchannel classification can be performed based on $X$. More specifically, a threshold of $x_{0}$ is chosen to mark sub-channels with $X \geq x_{0}$ as HQ sub-channels and those with $X<x_{0}$ as LQ sub-channels. The selection of $x_{0}$ must ensure that the ratio between the total sub-channel numbers of HQ and LQ sub-channel groups equals the rate ratio of the HP and LP layers. For example, if the rate ratio of the HP and LP layers is $1: 1, x_{0}$ should be the median of $X$.

The group membership of each sub-channel can be indicated by a single bit, for example, 1 for an HQ sub-channel and 0 for an LQ sub-channel. Then the classification of all the sub-channels can be represented by a bit vector, named sub-channel allocation vector (SCAV). The length of SCAV equals the number of sub-channels.

SCAV should be known and synchronized between the transmitter and receiver. At the transmitter, HP and LP layer data are allocated to $\mathrm{HQ}$ and LQ sub-channel groups according to SCAV; at the receiver, the recovery of the HP and LP layer data is also dictated by SCAV. Since the channel is time-variant, sub-channel classification and SCAV synchronization should be performed periodically. The selection of the time interval is a tradeoff between overhead and performance.

The sub-channel classification and SCAV generation are based on the channel estimation results. For a system in TDD mode, since the uplink (from receiver to transmitter) and the downlink (from transmitter to receiver) channels are reciprocal, there are two ASCA-UEP schemes: a receiverbased scheme and a transmitter-based scheme, which are detailed below. 


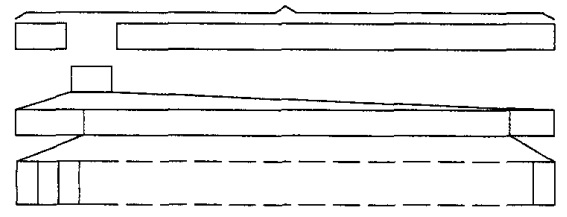

Fig. 1. Burst structure of the receiver-based ASCA-UEP scheme.

\section{B. Receiver-based Scheme}

In this scheme, channel estimation and sub-channel classification are performed at the receiver. Then SCAV is sent back to the transmitter through the feedback channel. Since the channel information is essential for STBC decoding, this scheme adds little extra complexity to the receiver.

Fig. 1 illustrates the frame structures over the downlink and uplink channels for the receiver-based scheme. Without loss of generality, propagation delay is assumed negligible. The transmitter starts a burst cycle by sending a pilot symbol, which is used for the receiver to perform channel estimation and synchronization. Based on the estimation results, SCAV is generated and sent back to the transmitter in a feedback burst. Under the instruction of SCAV, the transmitter starts a data burst for video data transmission by allocating HP (LP) data onto HQ (LQ) sub-channels. Each data burst lasts for a number of OFDM symbols to transmit an entire video data frame. Afterwards, a new cycle of operation begins with another pilot burst sent by the transmitter.

\section{Transmitter-based Scheme}

In this scheme, channel estimation is carried out at the transmitter based on the pilot sent by the receiver. If the receiver also adopts STBC over the uplink, there is no extra overhead since channel estimation is needed for STBC decoding at the transmitter. If there is no STBC over the uplink, the uplink pilot burst increases the overhead. However, as shown later, this scheme has better performance than the receiver-based scheme when the transmitter has sufficient power but the receiver is power-limited.

The frame structures of the transmitter-based scheme is shown in Fig. 2. The receiver starts a burst cycle by sending a pilot sequence for the transmitter to perform channel estimation and SCAV generation. Then SCAV is generated at the transmitter and delivered to the receiver, followed by a number of data symbols. Afterwards, the receiver starts a new cycle of operation by sending another pilot burst.

\section{Discussion}

In this section, we discuss the influence of transmission error on the receiver-based and transmitter-based schemes, and identify the suitable application environments for each scheme.

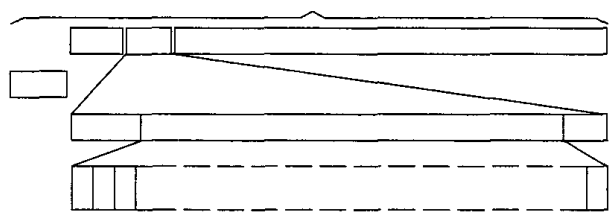

Fig. 2. Burst structure of the transmitter-based ASCA-UEP scheme.

Table 1

SIGNALING EXCHANGE OVER DOWNLINK AND UPLINK.

\begin{tabular}{c|c|c}
\hline & Downlink & Uplink \\
\hline \hline $\begin{array}{c}\text { Receiver-based } \\
\text { ASCA-UEP }\end{array}$ & $\begin{array}{c}\text { Pilot \& } \\
\text { Layered Video Data }\end{array}$ & SCAV \\
\hline $\begin{array}{c}\text { Transmitter-based } \\
\text { ASCA-UEP }\end{array}$ & $\begin{array}{c}\text { Pilot, SCAV \& } \\
\text { Layered Video Data }\end{array}$ & Pilot \\
\hline
\end{tabular}

Both the receiver-based and transmitter-based schemes require both ends to have the same knowledge on sub-channel classification. In other words, SCAV should be identical on both sides. The mismatch of the SCAV will render the allocated layered video data unrecoverable. Thus an errorfree transmission of SCAV is required in both schemes. This can be achieved by employing strong error correction schemes and/or a high transmission power. Obviously, it is preferred to send the error-sensitive SCAV from the side with sufficient power. On the other hand, channel estimation can also be inaccurate, especially when the pilot sequence does not have sufficient SNR. However, the accuracy of channel estimation is much less critical than errors in SCAV on the performance of the whole system. Channel estimation inaccuracy mainly influences the allocation of "boundary" sub-channels, i.e., the sub-channels with $X$ close to $x_{0}$, the classification threshold. In contrast, sub-channels that are "far" from the "boundary" are much less likely to be assigned to the "wrong" group, i.e., a sub-channel in a deep fade is unlikely to be designated as an HQ sub-channel.

Table 1 presents the signaling exchange over the downlink and uplink for the two schemes. The main difference is the transmission direction of SCAV and pilot. From the discussions above, we conclude that the receiver-based scheme is suitable for the case when the receiver has sufficient power, while the transmitter-based approach is preferred when the receiver is power-constrained and the transmitter has sufficient power.

\section{Simulation Results}

In this section, the performance of the proposed schemes is evaluated by computer simulations.

\section{A. System Parameters and Channel Model}

In the proposed system, each OFDM symbol has 64 sub-channels, of which 48 sub-channels are for data. Total 
Table 2

ITU-R M.1225 INDOOR B CHANNEL MODEL.

\begin{tabular}{c|c|c}
\hline Tap & $\begin{array}{c}\text { Relative Delay } \\
\text { (ns) }\end{array}$ & $\begin{array}{c}\text { Average Power } \\
\text { (dB) }\end{array}$ \\
\hline \hline 1 & 0 & 0 \\
\hline 2 & 100 & -3.6 \\
\hline 3 & 200 & -7.2 \\
\hline 4 & 300 & -10.8 \\
\hline 5 & 500 & -18 \\
\hline 6 & 700 & -25.2 \\
\hline
\end{tabular}

symbol duration is $4 \mu \mathrm{s}$. These parameters are identical to those of IEEE 802.11a [8]. QPSK is used as the modulation scheme and Almouti's STBC scheme [6] is employed.

The delay profile of indoor wideband channel model B (see Table 2), provided in ITU-R recommendation [9], is adopted for the simulation of uncorrelated multipath Rayleigh fading channels. The Doppler frequency is fixed at $100 \mathrm{~Hz}$, which corresponds to a moving speed of about $6 \mathrm{~m} / \mathrm{s}$ at the $5 \mathrm{GHz}$ band. The video data frame length is 10 OFDM symbols for all the schemes.

The standard video sequence 'Mobile' is used as the video source. This sequence is in the common intermediate format (CIF) with a resolution of $352 \times 288$, and a sampling ratio of $4: 2: 0$. The total data rate is fixed at $3 \mathrm{Mbps}$. For all two-layer schemes, the data rate ratio between HP and LP layers is fixed at $1: 1$. In all simulations, video concealment is not used.

\section{B. Simulation Results}

In this section, we present the performance evaluation results. The system performance in terms of the quality of the output video is measured by the peak signal-to-noise ratio (PSNR), given by

$$
P S N R=10 \log _{10}\left(\frac{255 \times 255}{M S E}\right),
$$

where

$$
M S E=\frac{1}{L V H} \sum_{l=1}^{L} \sum_{v=1}^{V} \sum_{h=1}^{H}\left[Y_{l}(v, h)-Y_{l, 0}(v, h)\right]^{2},
$$

in which $L$ is the total number of video frames, $V \times H$ is the video dimension, $Y_{l, 0}(v, h)$ is the pixel value of frame $l$ decoded with error-free data and $Y_{l}(v, h)$ is that of the reconstructed video at the receiver.

Besides the two ASCA-UEP schemes, we also evaluate two other schemes: a two-layer data partitioning scheme with FEC-based UEP and a single-layer scheme with no UEP. The FEC coding rates of the four schemes are listed in Table 3. They are chosen so that the total transmission data rates are identical for all schemes. The scheme index number will be used in the following discussion for simplicity.
Table 3

FEC CODING RATES OF DIFFERENT SCHEMES.

\begin{tabular}{c|c|c|c}
\hline Index & Scheme & $\begin{array}{c}\text { FEC rate for } \\
\text { HP Data }\end{array}$ & $\begin{array}{c}\text { FEC rate for } \\
\text { LP Data }\end{array}$ \\
\hline 1 & $\begin{array}{c}\text { Receiver-based } \\
\text { ASCA-UEP }\end{array}$ & $\frac{1}{2}$ & $\frac{1}{2}$ \\
\hline 2 & $\begin{array}{c}\text { Transmitter-based } \\
\text { ASCA-UEP }\end{array}$ & $\frac{1}{2}$ & $\frac{1}{2}$ \\
\hline 3 & FEC-based UEP & $\frac{1}{3}$ & No FEC \\
\hline 4 & $\begin{array}{c}\text { Single-layer Video } \\
\text { No UEP }\end{array}$ & \multicolumn{2}{|c}{$\frac{1}{2}$} \\
\hline
\end{tabular}

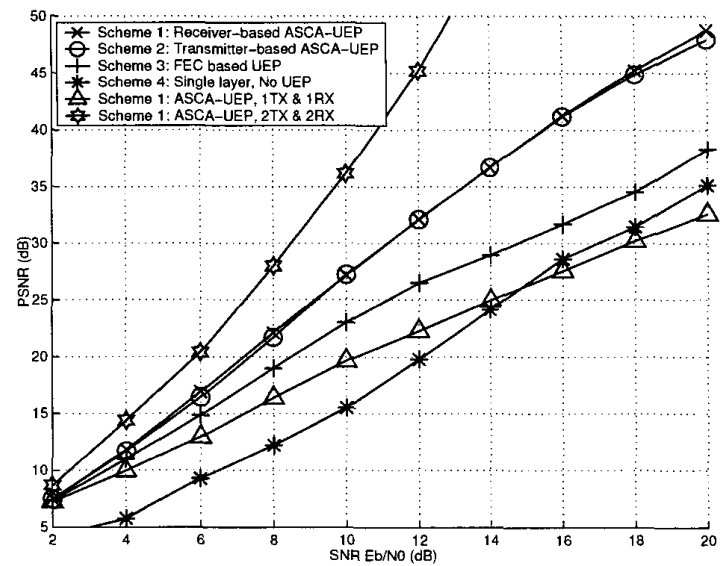

Fig. 3. PSNR performance of different schemes under ideal conditions.

1) Under Ideal Conditions: Fig. 3 presents the PSNR performance against $E_{b} / N_{0}$ for the four schemes under ideal situations, i.e., perfect channel estimation and errorfree transmission of SCAV. We observe that Schemes 1 and 2 have almost the same performance, and their performance is much better than that of Schemes 3 and 4 . The performance gap widens with the increase of SNR. At an SNR of $16 \mathrm{~dB}$, our proposed schemes have at least a PSNR gain of $10 \mathrm{~dB}$ compared to the other two schemes. Scheme 3 is also better than Scheme 4. This shows the ability of UEP in performance enhancement.

For reference, the performance of Scheme 1 with no diversity (single antenna at both sides) and Scheme 1 with two antennas at both sides are also shown in Fig. 3. We find that better performance can be obtained when more antennas are employed.

2) Receiver-based Scheme v.s. Transmitter-based Scheme: For the receiver-based scheme, as discussed in Sec. III, noise on the uplink introduces errors on SCAV. Since SCAV is critical, CRC is used for error detection together with powerful channel coding. If errors are detected in the received SCAV, the transmitter reuses the last correctly received SCAV for sub-channel allocation and informs the receiver. This method 


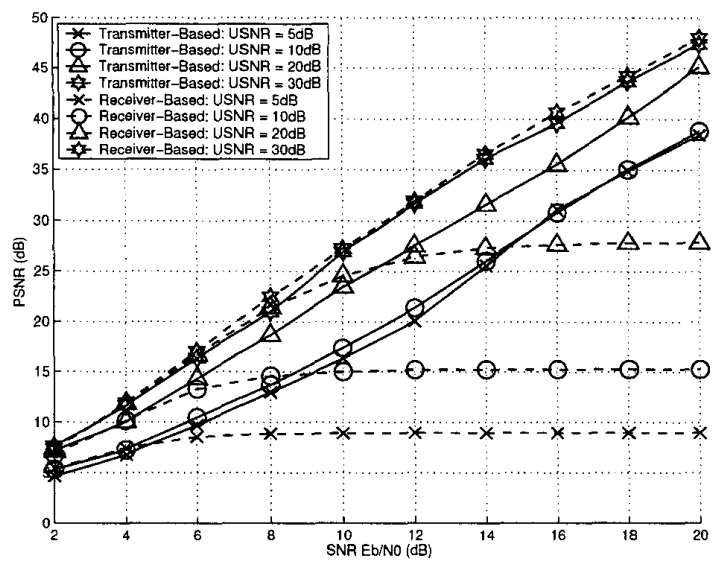

Fig. 4. PSNR performance of the receiver-based and transmitter-based schemes.

can keep the synchronization of SCAV between the two sides, but inevitably causes performance degradation due to time variations of the channel.

For the transmitter-based scheme, the SCAV transmission over the downlink channel is assumed error-free. This assumption is reasonable when the following conditions are met: 1) High transmission power is available over the downlink channel while the uplink transmission is power stringent; 2) Powerful channel coding is employed over the downlink. On the other hand, channel estimation at the transmitter is inaccurate since the pilot burst over the uplink does not have sufficient power. According to [10], the mean squared error (MSE) on an estimated time domain channel tap is $\frac{1}{\text { USNR }}$, where USNR is the uplink SNR on a pilot symbol.

Fig. 4 compares the performance of Schemes 1 and 2 for different SNRs and USNRs. It is obvious that the uplink channel noise level has severe impact on the receiver-based scheme. With low USNR, PSNR is quickly saturated with respect to SNR. For example, when USNR is lower than $20 d B$, PSNR is no higher than $28 d B$ regardless of SNR. Therefore, if we require PSNR to be higher than $30 \mathrm{~dB}$, USNR should also be higher than $20 \mathrm{~dB}$. In contrast, the transmitter-based scheme is hardly affected by uplink channel noise. PSNR always improves with the increase of SNR.

\section{CONCLUSION}

In this paper, we introduce the idea of ASCA-UEP to STBC-OFDM systems. By exploiting the reciprocity of TDD, we propose the receiver-based and transmitter-based schemes. The new schemes achieve better performance than the FEC-based UEP scheme with little additional complexity and overhead. Performance gain is even more significant when more receive antennas are employed. Furthermore, from analysis and simulation, we show that the transmitterbased scheme is more robust to uplink channel noise than the receiver-based scheme, and preferred when the receiver is power-constrained and the transmitter has sufficient power.

\section{ACKNOWLEDGMENTS}

This research is supported in part by the Research Grants Council of Hong Kong Special Administrative Region, China (Project no. HKU 7047/00E).

\section{REFERENCES}

[1] Y. Li, J. C. Chuang, N. R. Sollenberger, "Transmitter diversity for OFDM systems and its impact on highrate data wireless networks," IEEE J. Select. Areas Commun., Vol. 17, No. 7, pp. 1233-1243, July 1999.

[2] L. Hanzo, J. Streit, "Adaptive low-rate wireless video phone schemes," IEEE Trans. Circuits Syst. Video Technol., Vol. 5, No. 4, pp. 305-319, Aug. 1995.

[3] D.G. Daut, J.W. Modestino, "Two-dimensional DPCM image transmission over fading channels," IEEE Trans. Commun., vol. 31, No. 3, pp. 315-328, Mar. 1983.

[4] G.H. Yang, D. Shen, V.O.K. Li, "UEP for video transmission in space-time coded OFDM Systems," Proc. IEEE INFOCOM'04, Hong Kong, China, Mar. 2004.

[5] ISO/IEC 13818-2, Recommendation ITU-T H.262, 1995.

[6] S.M. Alamouti, "A simple transmit diversity technique for wireless communications," IEEE J. Select. Areas Commun., vol. 16, No. 8, pp. 1451-1458, Oct. 1998.

[7] V. Tarokh, H. Jafarkhani, A.R. Calderbank, "Spacetime block codes from orthogonal designs," IEEE Trans. Inform. Theory, vol. 45, No. 5, pp. 1456-1467, July 1999.

[8] IEEE Std 802.11a-1999, "Part 11: Wireless LAN medium access control (MAC) and physical layer (PHY) specifications: High-speed physical layer in the $5 \mathrm{GHz}$ band," ISO/IEC 8802-11:1999/Amd 1:2000(E), 1999.

[9] ITU-R Recommendation, ITU-R M.1225, "Guidelines for evaluation of radio transmission technologies for IMT-2000."

[10] Y. Li, N. Seshadri, S. Ariyavisitakul, "Channel estimation for OFDM systems with transmitter diversity in mobile wireless channels," IEEE J. Select. Areas Commun., vol. 17, No. 3, pp. 461-471, Mar. 1999. 